\title{
Approximate Truth vs. Empirical Adequacy
}

\begin{abstract}
Suppose that scientific realists believe that a successful theory is approximately true, and that constructive empiricists believe that it is empirically adequate. Whose belief is more likely to be false? The problem of underdetermination does not yield an answer to this question one way or the other, but the pessimistic induction does. The pessimistic induction, if correct, indicates that successful theories, both past and current, are empirically inadequate. It is arguable, however, that they are approximately true. Therefore, scientific realists overall take less epistemic risk than constructive empiricists.
\end{abstract}

Keywords: approximate truth, empirical adequacy, pessimistic induction, underdetermination

Park, Seungbae (2014). “Approximate Truth vs. Empirical Adequacy”, Epistemologia 37 (1): 106-118.

Seungbae Park

Ulsan National Institute of Science and Technology

Republic of Korea

nature@unist.ac.kr

\section{Introduction}

The cell theory, the Big Bang theory, the theory of electromagnetism, and the theory of thermodynamics are all successful scientific theories. A theory is said to be successful "if it makes substantially correct predictions, if it leads to efficacious interventions in the natural order, if it passes a battery of standard tests" (Laudan, 1981: 23). Laudan's definition of success captures the most important aspect of the aforementioned scientific theories, viz., some observational consequences of each of them have turned out to be true. What can we infer from the fact that some observational consequences are revealed to be true? Scientific realists ('realists' henceforth) and constructive empiricists ('empiricists' henceforth) have different answers to this question.

Suppose that realists infer that a successful scientific theory is approximately true, and that empiricists infer that it is empirically adequate. An interesting question arises: whose belief is more likely to be false? My answer is that the empiricist belief is more likely to be false than the realist one. My contention collides with what some philosophers say in the literature:

Certainly the realist takes an extra epistemic risk by believing the background theories to be (approximately) true rather than only empirically adequate. (Ladyman, Douven, Horsten, and van Fraassen, 1997: 308)

On a charitable interpretation, however, these philosophers mean truth, not approximate truth. After all, they are not in the context of adjudicating between approximate truth and empirical adequacy, but in the context of disputing the reliability of inference to the best explanation. They must be unreflectively following the standard practice of putting '(approximately)' in front of 'true.' That is, they used the qualifier 'approximately' without seriously reflecting on the contest between approximate truth and empirical adequacy. 
In this paper, I will explore in depth the issue of which notion involves more epistemic risk, approximate truth or empirical adequacy. I will argue that the problem of underdetermination does not come out in favor of either one over the other, but that the pessimistic induction does. The pessimistic induction, if correct, indicates that empirical adequacy is harder to come by than approximate truth. It will be shown that approximate truth withstands the gauntlet of the pessimistic induction better than empirical adequacy. My thesis may be surprising to those who casually think that we are epistemically safer, if we restrict our beliefs to observables.

\section{Ground Clearing}

Empiricists might argue that realists take a greater epistemic risk than empiricists because the content of the realist belief goes beyond that of the empiricist belief. Realists believe what a theory says about observables and unobservables whereas empiricists believe only what a theory says about observables. To put it another way, realists believe that a successful theory is approximately true, so they ipso facto believe that it is empirically adequate, whereas empiricists believe only that it is empirically adequate. Thus, realists believe what empiricists believe, plus they believe more. Believing more means a higher probability of error. Therefore, we can know a priori that the realist belief has a greater chance of being false than the empiricist belief. We do not need to consider the contest between the rivaling beliefs in the light of underdetermination and the pessimistic induction.

The foregoing argument, however, has a flaw. An approximately true theory is not necessarily empirically adequate. It describes unobservables incorrectly to some degree. The mischaracterization of unobservables may not affect observational consequences, in which case the theory is empirically adequate. But it may affect observational consequences to some degree, in which case the theory is approximately empirically inadequate. A theory can be said to be approximately empirically adequate, if all of its observational consequences are individually approximately true, or if "most of its observational consequences are true" (Park, 2009: 117, footnote). Thus, an approximately true theory is either empirically adequate or approximately empirically adequate. My contention would be endorsed by Mizrahi:

..mature scientific theories are approximately true, which seems to mean that the world should behave "exactly as if" those theories are true, or at least to a very high degree of 'as if', not "to some extent as if" they are true (or some other low degree of 'as if'). (Mizrahi, 2012: 136)

To say that the world behaves "exactly as if" a successful theory is true entails that it is empirically adequate. To say that the world operates "to a very high degree as if" a theory is true entails that it is approximately empirically adequate. Thus, when realists believe that a theory is approximately true, they may believe not that it is empirically adequate but that it is approximately empirically adequate.

Empiricists might insist that even if realists settle for approximate truth, the realist belief has more content than the empiricist belief because the realist belief concerns unobservables whereas the empiricist belief does not. The realist belief has the extra content that the empiricist belief does not have. Of course, the empiricist belief also has the extra content that the realist belief does not have, given that the content of the empiricist belief about observables goes beyond the content of the realist belief about observables. But the extra realist content is greater than the extra empiricist content because the extra realist content involves unobservables whereas the extra empiricist content involves observables. Therefore, the realist belief is more likely to be false than the empiricist belief. 
The foregoing empiricist argument is suspect. The premise that the extra realist content involves unobservables whereas the extra empiricist content involves observables does not justify the conclusion that the extra realist content is greater than the extra empiricist content. An additional premise is required for the argument to be convincing. After all, unobservables do not have the magical power that makes the content of a human belief about them greater than the content of a human belief about observables. An example would be useful to support this abstract point.

Suppose that a scientific theory consists of five theoretical statements. When conjoined with auxiliary assumptions, they entail infinitely many observational consequences. Realists believe that four of the five theoretical statements are true, and that $90 \%$ of the infinitely many observational consequences are true. In contrast, empiricists believe that $100 \%$ of the infinitely many observational consequences are true. Whose belief has a greater content? It is not clear what the correct answer is. Suppose that $10 \%$ of all the infinitely many observational consequences are over four million observational statements. There is no guarantee that the contents of over four million observational statements are smaller than the contents of the four theoretical statements.

The point of the preceding example is to show that it is difficult to compare the contents of two beliefs if one belief does not entail the other belief. Given that the realist belief does not entail the empiricist belief, it is difficult to determine $a$ priori that the content of the realist belief is greater than the content of the empiricist belief, and hence the realist belief is more likely to be false than the empiricist belief. For this reason, I turn to the problem of underdetermination and the pessimistic induction.

\section{Adjudication}

\subsection{The Problem of Underdetermination}

Consider van Fraassen's famous example of underdetermination (1980: 46). Newton's theory of motion consists of the law of inertia, $\mathrm{F}=\mathrm{ma}$, the law of action-reaction, $\mathrm{F}=\mathrm{Gm} \mathrm{m}_{1} \mathrm{~m}_{2} / \mathrm{r}^{2}$, and the postulate that the gravitational center of the solar system is at absolute rest. We can generate an infinite number of competing theories by varying the absolute velocity of the center. They are all empirically equivalent to each other, i.e., they make the same claims about observables. They are, however, incompatible with each other because they make different claims about the absolute velocity of the gravitational center which is unobservable. It appears that observation cannot determine which of them is true, and that we are not justified in believing that Newton's theory of motion is true.

Suppose that realists believe that Newton's theory of motion is approximately true, and that empiricists believe that it is empirically adequate. Who take a greater epistemic risk? It is not clear what the correct answer is. Initially, it seems that realists take the greater epistemic risk because they believe what the theory says about unobservables. As Psillos (1997: 370) points out, however, from the fact that rival theories are empirically equivalent to each other, it does not necessarily follow that they are empirically adequate. They might be far less than empirically adequate. Thus, an argument is required to move from empirical equivalence to empirical adequacy, and empiricists take some epistemic risk when they infer that Newton's theory of motion is empirically adequate.

Empiricists might argue that Newton's theory of motion is empirically adequate under the assumption that one of the rival theories is true. After all, a theory empirically equivalent to a true theory is necessarily empirically adequate. Therefore, the empiricist belief is true. Realists would reply, however, that if one of the rival theories is true, the rest of them "are all approximately true because they share the important assumptions: the three laws of motion 
and the law of gravity" (Park, 2001: 123). Therefore, van Fraassen's example of underdetermination does not support the claim that empirical adequacy is more obtainable than approximate truth or vice versa. As it stands, we do not know which one is more procurable.

Let me turn to the example of quantum mechanics. Bohm's version of quantum mechanics is empirically equivalent to von Neumann and Dirac's version of quantum mechanics, but they differ radically in their claims about unobservables. The former claims that micro-events are determinate just like macro-events, and that there exists something that can travel faster than light, while the latter claims that unlike macro-events, micro-events are indeterminate, and nothing travels faster than light. In short, the two versions make radically different claims about unobservables. Accordingly, we are not justified in believing that von Neumann and Dirac's version is approximately true. It is reasonable, however, to believe that it is empirically adequate. Therefore, empirical adequacy is epistemically closer to us than approximate truth is. So it seems.

However, if the two versions of quantum mechanics are not even approximately true, it is not clear how they are so successful in predicting phenomena. In general, a completely false theory does not make substantially true predictions. How can a theory which makes completely false claims about unobservables nonetheless pass the battery of standard tests? As Putnam (1975: 73) puts it, it would be a miracle if a completely false theory were successful. Thus, we have two different intuitions pulling in opposite directions. It is controversial whether the two versions of quantum mechanics are approximately true or completely false.

It is also controversial whether the two versions of quantum mechanics are empirically adequate or inadequate. As we previously noted, from the fact that rival theories are empirical equivalent, it does not necessarily follow that they are empirically adequate. After all, empirically inadequate theories can be empirically equivalent to each other. From the fact that the two versions of quantum mechanics are successful, it does not necessarily follow that they are empirically adequate. Success involves the truth of some observational consequences, whereas empirical adequacy involves the truth of all observational consequences. An argument is required to infer from some to all. In the absence of an argument, it is simply an open question whether the two versions are empirically adequate or inadequate. We cannot rule out the possibility that they are approximately empirically adequate or far less than approximately empirically adequate. Therefore, realism is no worse off than antirealism visà-vis the underdetermination of quantum mechanics.

Both the underdetermination of Newtonian mechanics and that of quantum mechanics are inert as arbiters between approximate truth and empirical adequacy. In discussing the former, I assumed that one of the rival theories is true. In discussing the latter, in contrast, I did not make such an assumption. I operated only under the premises that the two rival versions of quantum mechanics are empirically equivalent, and that they are equally successful. In both cases of underdetermination, empirical adequacy did not come out as a winner over approximate truth. This conclusion would be endorsed by Leplin (1997) and Stanford (2000).

Leplin and Stanford argue that when a true theory competes with a false theory, there is bound to be structural similarity between them beyond observational similarity. The structural similarity ensures that the false theory is approximately true:

..false, successful theories bear some connection or similarity to the true one beyond predictive success, which explains their predictive success. (Leplin, 1997: 14) 
..any two theories that make similar predictions over a domain of any significant extent, there is sure to be something we could fasten onto as a structural similarity or isomorphism between them. (Stanford, 2000: 274)

If Leplin and Stanford are right that a false theory competing with a true theory is bound to be approximately true, it is clear that underdetermination does not favor empiricism over realism, i.e., that underdetermination does not yield the reason for thinking that approximate truth is epistemically farther from us than empirical adequacy.

\subsection{The Pessimistic Induction}

Unlike the problem of underdetermination, the pessimistic induction, if correct, does show that approximate truth is more attainable than empirical adequacy. The pessimistic induction asserts that successful present theories are false because successful past theories are false:

The ephemeral nature of scientific theories takes by surprise the man of the world. Their brief period of prosperity ended, he sees them abandoned one after the other; he sees ruins piled upon ruins; he predicts that the theories in fashion today will in a short time succumb in their turn, and he concludes that they are absolutely in vain. (Poincaré, 1905/1952: 160)

It is well-known in the literature that the pessimistic induction poses a serious threat to the realist quest for truth, but it is not widely appreciated that the pessimistic induction poses an equal threat to the empiricist aspiration to empirical adequacy:

The successful past theories turned out to be empirically inadequate. So successful current theories will turn out to be empirically inadequate as well. (Park, 2001: 78)

Most of these theories eventually turned out not to be empirically adequate. Therefore, we should believe that probably, most of the theories we currently accept are not empirically adequate either. (Lange, 2002: 282)

Successful past theories, such as the phlogiston theory of combustion and the caloric theory of heat, turned out to be false when they ran into anomalies, which implies that they were disclosed to be empirically inadequate. Thus, the pessimistic induction refutes the empiricist position that a successful theory is empirically adequate.

Does the pessimistic induction also devastate the realist position that a successful theory is approximately true? If Kitcher and Psillos are right, the answer is no. Kitcher (1993: 140-149) argues that the ether theory might be approximately true, given that its working posits are true, although its idle posits are false, in the light of current theories. From the historical fact that working posits of the caloric theory of heat were carried over to the kinetic theory of heat, and from the historical fact that the most eminent caloric theorists were not committed to the truth of the idle posits of the theory, Psillos concludes that "it makes perfect sense to talk of the approximate truth of this theory [the caloric theory of heat]" (1999: 113).

Antirealists' critical response to Kitcher's approach and Psillos's approach to the pessimistic induction in the literature is to argue that it is hard to distinguish between idle and working assumptions of current theories, although it is relatively easy to discriminate between idle and working assumptions of past theories in retrospect. Stanford puts the problem as follows: 
The problem with this strategy, of course, is that at the time of our commitment to a theory it is not usually possible to separate its operative elements from the extra baggage (assuming that this separation is coherent at all), as Maxwell's famous remark (paraphrased in Laudan 1981, 114 ) that 'the aether was better confirmed than any other theoretical entity in natural philosophy' reminds us. (Stanford, 2000: 278, footnote)

If Maxwell is right, the ether theorists were confident of the truth of those elements of the ether theory that turned out to be idle. This goes against realists' presupposition that scientists can isolate the idle elements of a current theory and can avoid assenting to their truths.

Let me make a few comments about Stanford's critical response to Kitcher and Psillos. Firstly, Kitcher makes an important point about Maxwell's remark as reported by Laudan, viz., "Maxwell was wrong" (1993: 149). His claim is based on his detailed case study on the ether theory, which I will not rehearse here. The upshot of his case study is that the working posits of Fresnel's ether theory are "endorsed by contemporary physics" (1993: 145), that "Fresnel typically makes no detailed claims about the nature of this medium [ether]" (1993: 147), and that the success of the ether theory provided support only for the working posits.

Secondly, Stanford's criticism, even if correct, does not knock down Kitcher and Psillo's suggestion that working parts are distinguishable from idle parts. All it shows is that realists need to do more work to come up with a principled distinction between idle assumptions and working assumptions. I (2011: 23-29) have recently attempted to improve Kitcher and Psillos's criteria. Let me briefly introduce here a criterion to recognize a working posit. In certain cases in science, a constituent of $\mathrm{T}_{1}$ and a constituent of $\mathrm{T}_{2}$ jointly explain a third phenomenon. Both $T_{1}$ and $T_{2}$ have their own observational evidence. Neither $T_{1}$ nor $T_{2}$ can explain the third phenomenon alone; they have to work together to explain it. In such cases, the constituent of $\mathrm{T}_{1}$ and the constituent of $\mathrm{T}_{2}$ are working posits and hence they will survive scientific revolutions in the future.

Thirdly, and the most important of all, even if there is no principled distinction between working and idle assumptions, we can say that successful past theories are approximately true. Suppose that the ether theory was comprised of ten theoretical claims: seven of them were carried over to its corresponding current theory, but three were not. We can then say that the ether theory was approximately true in the current light, even if we cannot find the common features of the seven carried-over theoretical claims and the common features of the three defunct theoretical claims. What counts is not whether or not we can separate operative elements from extra baggage but whether or not a theory is close to the truth. A theory can be said to be close to the truth in the light of its subsequent theory, if a significant portion of the theory survives a scientific revolution. Our inability to tell exactly which elements will be retained and which will be discarded does not prevent us from attributing approximate truth to a theory. In contrast, we cannot say that the successful past theories are empirically adequate; after all, they clearly ran into anomalies. Therefore, the empiricist belief that a successful theory is empirically adequate is more likely to be false than the realist belief that it is approximately true.

Laudan (1981) would object that successful past theories cannot even be approximately true, even if their working assumptions are true, because their central terms do not refer. The phlogiston theory and the ether theory, for instance, are completely false because the key terms 'phlogiston' and 'ether' do not refer. In other words, since the successful past theories are about nonexistent objects, they cannot even be approximately true.

In response, Kitcher (1993: 75-105) develops a theory of reference that the reference of a token of a scientific term is fixed in three ways: the descriptive mode, the baptismal mode, and the conformist mode. The terms, 'phlogiston' and 'dephlogisticated air,' fail to refer, if 
their references are fixed solely by the descriptive mode of reference, because there is no substance emitted in combustion. On some occasions, however, phlogiston theorists use the baptismal mode of reference or the conformist mode of reference to pick out an object. On those occasions, 'dephlogisticated air' refers to oxygen, and their assertions about the referent of 'dephlogisticated air' are true (Kitcher, 1993: 100-102). In short, in some contexts 'dephlogisticated air' refers to oxygen, and phlogiston theorists made true assertions about oxygen.

Psillos (1999, Chapter 12) develops his own theory of reference the gist of which is that a theoretical term refers to a theoretical entity in virtue of the causal relation between "kind-constitutive properties" of the theoretical entity and "core causal descriptions" associated with the theoretical term. A kind-constitutive property is a property that makes an object belong to a kind. A core causal description is a description of a kind-constitutive property; it has its causal origin in the kind-constitutive property. The core causal descriptions of the ether theory are descriptions of the properties of a putative entity that cause light phenomena. Psillos argues that 'ether' refers to the electromagnetic field "because the core causal description associated with the term 'electromagnetic field' takes up the core causal description associated with the term "ether"' (1999: 296).

My response to the problem of reference is to argue like Psillos (1999: 294) that the key terms of the past theories approximately refer to the referents of the key terms of their corresponding present theories. For example, 'phlogiston' and 'ether' approximately refer to oxygen and electromagnetic field. The justification for this assertion is that the past theories and the present theories share assumptions. On this account, reference is determined by description and is not an all-or-nothing affair. Past theoretical terms are not complete referential failures.

\section{Objections and Replies}

Empiricists might admit that the pessimistic induction hits the empiricist belief harder than the realist belief, but they might complain that the realist belief is untestable, given that it concerns unobservables and allows anomalies. Even if a theory clashes with some phenomena, realists may stick to their belief that it is approximately true. Since the realist belief is untestable, it is worthless to hold it. In contrast, the empiricist belief is testable. If a theory collides with some phenomena, it turns out to be empirically inadequate, so empiricists would give up their belief that it is empirically adequate. Since the empiricist belief is testable, it is worthwhile to hold it.

Let me use an analogy to illuminate the difference between the realist belief and the empiricist belief in terms of testability. Suppose that there are two cats, one is inside a black box, and the other is outside the black box. Realists believe that the cat inside the black box is alive, and empiricists believe that the cat outside the black box is alive. They can observe the cat outside the black box, but not the cat inside the black box. It turns out that the cat outside the black box is dead. The realists claim that in such circumstances, the empiricist belief is more likely to be false than the realist belief because the empiricist belief does not even have a chance to be true, whereas the realist belief has. The empiricists complain that we cannot tell whether the cat inside the black box is alive or dead, so it is worthless to hold the realist belief that the act inside the black box is alive. In contrast, it is worthwhile to hold the empiricist belief that the cat outside the black box is alive.

My reply to the preceding possible complaint from empiricists is twofold. First, cherishing testability at the cost of holding false beliefs comes with a heavy price, viz., we may form all sorts of absurd false beliefs, such as snow is black, and the earth is flat. These 
provocative beliefs are all false but testable, so the empiricists would say that it is worthwhile to hold them. Realists would disagree, pointing out that we enshrine testability because we want to avoid false beliefs, i.e., testability is a means to achieve the end of avoiding false beliefs. To embrace false beliefs for the sake of testability is to discard the end to achieve the means.

Second, it is merely an assumption that the realist belief is untestable while the empiricist belief is testable. An argument is required to justify the assumption. The argument cannot appeal to underdetermination because as we previously noted, underdetermination does not show that we have a better epistemic access to empirical adequacy than to approximate truth. Moreover, realists would protest that their belief is testable. Approximate truth entails approximate empirical adequacy, so a theory is revealed to be completely false, if most of its observational consequences are disclosed to be false.

Empiricists might insist that the realist belief is untestable on the ground that we can never ascertain the truths or the falsities of most of observational consequences of a theory. The observational consequences are claims about observable events that occurred, occur, and will occur, so we can never obtain all the relevant observational data that would reveal that most observational consequences of a theory are true or false. Therefore, we can never know whether a theory is approximately true or completely false.

Let me point out, however, that the standard of testability implicit in the foregoing criticism is too high. If the realist belief is not testable because we cannot obtain observational data corresponding to most of observational consequences of a theory, the empiricist belief is not testable either because we cannot obtain observational data corresponding to all observational consequences of a theory. It is trivially true that it is harder to establish empirical adequacy than approximate empirical adequacy.

Empiricists might now launch a semantic objection against realism, viz., the notion of approximate truth is obscure. It is not clear how close a theory must be to the truth in order to be counted as being approximately true. I admit that approximate truth is not a precise notion, and there is a huge literature on the definition of approximate truth. I can only defer exploring this territory to a future occasion. Let me make a brief point, though, that is not appreciated in the literature. Empiricists need the notion of approximate truth too. When scientists perform an experiment correctly, there might be a gap between an expected outcome and an actual outcome. In such cases, empiricists may have to say that the relevant observational consequence is approximately true as opposed to exactly true.

In order to defend realism, I have piggybacked on the empiricist inference from success to empirical adequacy and on the empiricist use of the concept of approximate truth. In order to diffuse my strategy, empiricists may now embrace skepticism. Skepticism in this context means the refusal to infer anything from the success of a scientific theory. Skeptics do not go beyond the belief that some observational consequences of a successful theory are true. They do not even believe that a successful theory is empirically adequate. They only challenge realists to justify their belief.

A problem of skepticism in the context of the debate between realists and empiricists is that it is off-limit as the following philosophers eloquently put it:

Skepticism is an ugly threat; a philosophical position which leads to skepticism reduces itself to absurdity. (Ladyman, Douven, Horsten, and van Fraassen 1997: 317)

Kitcher would agree with these philosophers, for he says that "Skeptics who insist that we begin from no assumptions are inviting us to play a mug's game" (1993: 135). Both realism 
and empiricism are built upon the assumption that we can go beyond the belief that some observational consequences of a successful theory are true. In order to avoid skepticism, empiricists must stick their necks out and infer something from the fact that a theory is successful.

\section{Conclusion}

A moderate position and an ambitious position are available for both realists and empiricists. The moderate realist position asserts that successful theories are approximately true; the ambitious realist position, that they are exactly true. The moderate empiricist position maintains that successful theories are approximately empirically adequate; the ambitious empiricist position, that they are empirically adequate. This paper makes it clear, I hope, that the ambitious empiricist position is epistemically less secure than the moderate realist position in the light of the pessimistic induction, although the problem of underdetermination does not decide the issue one way or the other. Thus, being restricted to observational beliefs does not guarantee better epistemic security.

What if empiricists strive for approximate empirical adequacy, and realists for approximate truth? Of the two positions, which one should we choose? Clearly, it is harder to obtain approximate truth than approximate empirical adequacy because approximate truth entail approximate empirical adequacy. In my view, however, better epistemic security is the only advantage of empiricism over realism, while there are pragmatic disadvantages of empiricism that are not discussed in this paper (Park, 2014, Section 6). Moreover, there are pragmatic advantages of realism which outweigh the epistemic advantage of empiricism. Accordingly, the realist position is overall better than the empiricist position. Unfortunately, other studies are needed to flesh out and defend this bold line of reasoning.

\section{References}

Kitcher, Philip (1993). The Advancement of Science: Science without Legend Objectivity without Illusion. New York: Oxford University Press.

Ladyman, James, Igor Douven, Leon Horsten, and Bas van Fraassen (1997). A defense of van Fraassen's critique of abductive inference: Reply to Psillos. The Philosophical Quarterly 47 (188): 305-321.

Lange, Marc (2002). Baseball, pessimistic inductions and the turnover fallacy. Analysis 62 (4): 2881-85.

Laudan, Larry (1981). A confutation of convergent realism. Philosophy of Science 48 (1): 1949.

Leplin, Jarrett (1997). A novel defense of scientific realism. New York: Oxford University Press.

Mizrahi, Moti (2012). Why the ultimate argument for scientific realism ultimately fails. Studies in History and Philosophy of Science 43 (1): 132-138.

Park, Seungbae (2001). Scientific realism vs. scientific antirealism. Ph.D. Dissertation. 
University of Arizona.

(2009). Philosophical responses to underdetermination in science. Journal for General Philosophy of Science 40 (1): 115-124.

(2011). Coherence of our best scientific theories. Foundations of Science 16 (1): 2130. (2014) Accepting our best scientific theories. Pensée. 76 (3): 131-139.

Poincaré, Henri (1905/1952). Science and hypothesis. New York: Dover.

Psillos, Stathis (1997). How not to defend constructive empiricism: A rejoinder. The Philosophical Quarterly 47: 369-372.

(1999). Scientific realism: How science tracks truth. New York: Routledge.

Putnam, Hilary (1975). Mathematics, Matter and Method (Philosophical Papers, vo. 1), Cambridge: Cambridge University Press.

Stanford, Kyle (2000). An antirealist explanation of the success of science. Philosophy of Science 67: 266-284.

van Fraassen, Bas (1980). The scientific image. Oxford: Oxford University Press. 